\title{
Nivel de cumplimiento de los registros de Enfermería en el servicio de Ginecoobstetricia del Hospital Nacional Madre Niño San Bartolomé, 2012
}

\author{
Level of compliance records of Nursing in Obstetrics and Gynecology Service \\ National Mother Child Hospital San Bartolomé, 2012
}

Julia Virginia Gutiérrez Cahuana¹, Olga Yolanda Esquén Sembrera², Eleana Patricia Gómez Taguchi²

\begin{abstract}
RESUMEN
Objetivo: Determinar el nivel de cumplimiento de los registros de Enfermería con respecto a los cuidados que se brinda en el servicio de Ginecoobstetricia del HONADOMANI "San Bartolomé". Metodología: Es un estudio de tipo no experimental, descriptivo transversal. La población de estudio estuvo constituida por 87 historias clínicas de pacientes hospitalizadas en el servicio de Ginecoobstetricia durante la segunda semana del mes de abril del año 2012, obteniéndose una muestra de 41. Se elaboró una hoja de check list y se aplicó a los cuatro registros de Enfermería (Hoja de Funciones Vitales, Kárdex, Anotaciones de Enfermería y Hoja de Balance Hídrico) de las historias clínicas que fueron seleccionadas al azar. Resultados: Se encontró que las enfermeras del servicio de Ginecoobstetricia registran las notas de Enfermería entre bueno y muy bueno en un 75.6\%, registran las hoja gráfica entre bueno y muy bueno en un $95.1 \%$, al igual que el Kárdex en un $65.9 \%$. Mientras que la hoja de Balance Hídrico lo registran muy mal en un 65.9\%. Conclusión: Las enfermeras del servicio de Ginecoobstetricia, cumplen con realizar los registros de Enfermería entre un nivel bueno y muy bueno en $63.4 \%$ y el $7.3 \%$ registran muy malo.
\end{abstract}

Palabras clave: Registros de enfermería, nivel de cumplimiento.

\begin{abstract}
Objective: To determine the level of compliance of nursing records regarding the care that is provided in the service of Gynecology and Obstetrics HONADOMANI "San Bartolome". Methodology: It was a study of nonexperimental, descriptive transversal. The study population consisted of a population of 87 medical records of patients hospitalized in the service of Gynecology Obstetrics during the second week of April of 2012, yielding a sample of 41. A road check list was developed and applied the four nursing records (Sheet Vital Functions, Kardex, Annotations of Nursing and Water Balance Sheet) of medical records that were selected randomly. Results: It was found that the nurses of Obstetrics and Gynecology nursing notes recorded between good and very good in $75.6 \%$, recorded the chart sheet between good and very good in $95.1 \%$, as the Kardex in $65.9 \%$. While the Water Balance sheet as badly recorded a $65.9 \%$ Conclusion: Nurses ginecoobstetricia service, meet nursing records make between good and very good level in $63.4 \%$ and $7.3 \%$ recorded very bad.
\end{abstract}

Keywords: Nursing records, compliance.

${ }^{I}$ Jefa del Servicio de Ginecoobstetricia del Hospital Nacional Madre Niño San Bartolomé HONADOMANI, Lima.

${ }^{2}$ Enfermera asistencial Servicio de Ginecoobstetricia del Hospital Nacional Madre Niño San Bartolomé HONADOMANI, Lima.

Correspondencia: vigutierrez16@hotmail.com; patriciatagu@yahoo.es 


\section{INTRODUCCIÓN}

El registro de la actividad de la enfermera, en hospitalización, está basado en el Proceso de Atención de Enfermería (PAE), por considerar que es un método ordenado y sistemático para obtener información e identificar los problemas del individuo, la familia y la comunidad. Su fin es planificar, ejecutar y evaluar los cuidados de Enfermería (García, Navío y Valentín, 2007).

Los registros de Enfermería son instrumentos básicos del ejercicio del profesional de Enfermería, que presentan una visión completa y global del paciente que a futuro, que sirven como documentación legal de prácticas realizadas. Las razones de su utilización han evolucionado desde el tiempo en que Florence Nightingale los introdujo como instrumentos que la enfermera debía utilizar para atender a los pacientes. La documentación utilizada en aquella época recogía, principalmente, el cumplimiento de las órdenes del médico. La prestación de cuidados de Enfermería se realizaba "sobre la marcha", a medida que iban surgiendo las necesidades, aunque siempre existió una cierta programación de las actividades que atendía el seguimiento de horarios establecidos con registros mínimos o inexistentes. Actualmente las enfermeras afrontan una serie de dificultades en relación con los registros de Enfermería. Pues la mayoría de ellos no contienen la calidad de información requerida.

El registro se considera como un documento que avala la calidad, la continuidad de los cuidados, la mejora de la comunicación, evita errores o posibles duplicidades que pueden producirse en el servicio. Además, es un respaldo legal frente a posibles demandas y a la vez permiten evaluar retrospectivamente la calidad de los cuidados. (Amezcua, 1995; citado por Hernández, Rodríguez, Gutiérrez y Bolaños, 2007).

González (1999), manifiestan que los registros de Enfermería constituyen la evidencia de la intervención profesional de la evolución del paciente, el planeamiento del cuidado y la continuidad de la prestación. Además, es fuente de datos para la evaluación del cuidado y el proceso de la mejora continua e investigación.

(González e Ibarra, 2007; citado por Avendaño, Ramírez, Colín, Rentería y López 2011) refieren que las anotaciones de Enfermería son variadas, complejas y requieren tiempo pues las enfermeras pasan entre 35 y 140 minutos escribiendo, por turno. Como resultado de todo ello, con mucha frecuencia quedan sin anotar importantes observaciones y diálogos específicos, por falta de tiempo.
Córcoles, García, Ruiz, Pita y Callejas (2005), refieren que los profesionales de Enfermería no pueden seguir trabajando de forma intuitiva, permitiendo que la información sobre el trabajo de Enfermería se ignore por no quedar plasmado en registros adecuados. Esta afirmación responde a razones fundamentadas y a las necesidades de la sociedad actual; basta recordar que en atención especializada se trabaja por turnos, de manera que cada usuario es atendido por varios profesionales cada día, lo que hace imprescindible que exista registro de las actividades realizadas para dar continuidad a los cuidados y evitar tanto la omisión como la duplicidad de acciones. Los registros de Enfermería forman parte de la historia clínica aunque, específicamente, la jurisprudencia no determina las normas para estos registros, sino que emiten un juicio crítico sobre el cumplimiento de la norma. Otra razón, no menos importante, es que los registros de las actividades propias de Enfermería proporcionan información para la formación de pregrado, posgrado y la investigación.

La Ley del Trabajo del Enfermero N. ${ }^{\circ} 27669$, en el artículo N. 7 inciso (a), promulgada el 15 de febrero del año 2002, señala como responsabilidad de la enfermera: "Brindar cuidado integral de Enfermería basado en el Proceso de Atención de Enfermería (PAE)”.

El Colegio de Enfermeros del Perú promueve el trabajo en base al Proceso de Atención de Enfermería, lo que generará la implementación de nuevos registros de Enfermería como un medio para mantener la calidad y la continuidad de los cuidados de acuerdo a cada especialidad. Es así que en las Normas de Gestión del Cuidado Enfermero indican que los registros de Enfermería deberán proporcionar el conocimiento e informaciones necesarios que faciliten la labor de los profesionales y garanticen al paciente una asistencia, información y comunicación efectiva, pronta y segura.

Asimismo, Potter \& Perry (2005) señalan que: “El registro que realiza el personal de Enfermería, sobre el paciente, constituye un medio de comunicación. La documentación de Enfermería continúa evolucionando, viéndose incrementada su importancia”.

Aunque no existen muchas investigaciones disponibles sobre la calidad de los Métodos de Registros de Enfermería, existen trabajos como el de (Junttila, Salantera y Hupli, 2000; citado por Agustín 2008) donde se formula un método de registro para la documentación preoperatoria, con una terminología común. 
El estudio realizado por (Caballero, Becerra y Latrach, 2005; citado por Agustín 2008) demostraron que la mayoría del personal de Enfermería no aplica el proceso, porque no son factibles los métodos de registros. Asimismo, en la investigación realizada por (Loza y Vásquez, 1999; citado por Agustín 2008) en España, detectaron que $64 \%$ de los profesionales de Enfermería, consideraron difícil la aplicación del proceso, por ser complejo el método de registro utilizado y no quedar en ellos claro los pasos lógicos del PAE.

Sin embargo, en Cuba la aplicación del Proceso de Atención de Enfermería repercutió positivamente en la calidad de los registros de los enfermeros en las historias clínicas y en la propia producción científica.

García y Meza (2003) refieren que cada centro hospitalario construye sus propios modelos de registros, adaptados a sus necesidades. A pesar de la variedad, existen documentos comunes en su finalidad $\mathrm{y}$, aunque puedan tener distintos diseños, deben cumplir con las normas de la OMS como: los registros deben ser permanentes, con limitaciones, tener una identificación que permita su recuperación y localización cuando sea necesario y tener continuidad.

Para garantizar la gestión del cuidado es necesario contar con un plan de monitoreo de los registros de enfermería definido como el seguimiento continuo de las actividades relacionadas con la calidad de la oferta.

La Joint Comissión on Acreditation of Health Care Organizations (1991), recomienda el uso de indicadores clínicos verifican el cumplimiento de los registros.

En el Perú los registros de Enfermería contienen un conjunto de información sobre el progreso del paciente y forman parte de la documentación de la historia clínica, sin embargo se puede observar que existe una diversidad de registros, que cada institución ha ido adecuándolos a su realidad.

En este contexto, los cuidados de Enfermería son parte sustancial en la atención de salud y, por este motivo, influyen decisivamente en la calidad global que brinda una institución. Sin embargo, es a veces difícil ponderar el impacto de los cuidados de Enfermería, por lo que se hace necesario realizar medición a través de una auditoría de las acciones profesionales.

En el Hospital Nacional Docente Madre Niño "San Bartolomé", a raíz de los resultados obtenidos en la auditoria de las historias clínicas realizadas en el año 2010, el Departamento de Enfermería y el Comité de Historias Clínicas han elaborado y validado nuevos formatos de registros de Enfermería los cuales han sido estandarizados para ser aplicados a nivel de todos los servicios de hospitalización como son: Pediatría, Cirugía Pediátrica y Ginecoobstetricia.

En el servicio de Ginecoobstetricia la implementación de estos nuevos registros originó una serie de discrepancias y resistencia al cambio por parte de las enfermeras. Por lo que se planteó como objetivo general de investigación: Determinar el nivel de cumplimiento de los registros de Enfermería respecto a los cuidados que se brinda en el servicio de Ginecoobstetricia del HONADOMANI "San Bartolomé".

\section{MATERIAL Y MÉTODOS}

El estudio es de tipo no experimental, retrospectiva, descriptivo transversal que se realizó en el servicio de Ginecoobstetricia del HONADOMANI "San Bartolomé", utilizando un total de 87 historias clínicas de pacientes posoperadas atendidas en la segunda semana del mes de abril del 2012 a las cuales se aplicó el cálculo de muestra, obteniéndose 41 historias clínicas que fueron seleccionada aleatoriamente.

Los criterios de inclusión fueron: Historias clínicas de pacientes posoperadas de Ginecoobstetrícia, historias clínicas de Ginecoobstetricia, comprendidas entre la segunda semana del mes de abril del año 2012.

Se aplicó una hoja de verificación con 52 ítems elaborada por las autoras, distribuidas en 12 ítemes para los registros de las notas de Enfermería, 16 ítemes para hoja gráfica, 12 ítemes para balance hídrico y 12 ítemes para la hoja de Kardex; teniendo como base las normas de Gestión del Cuidado Enfermero (CEP). Esta hoja de verificación fue validada por las enfermeras supervisoras del HONADOMANI "San Bartolomé" , la Jefa del Servicio de Ginecoobstetricia y la coordinadora de Proyectos de la Oficina de Calidad del hospital.

El proceso de recolección de datos, así como el procesamiento y análisis estadísticos, fue ejecutado íntegramente por el equipo investigador, haciendo uso del programa estadístico SPSS versión 15 y luego organizada en tablas. 


\section{RESULTADOS}

\section{Tabla 1}

Consolidado del nivel de cumplimiento por registro de Enfermería en el servicio de Ginecobstetricia del HONADONANI "San Bartolomé", Lima- 2012.

\begin{tabular}{cccc}
\hline Indicadores & Frecuencia & & $\%$ N de la columna \\
\hline Cumplimiento del registro de & Muy malo & 2 & $4.9 \%$ \\
las Notas de Enfermería & Malo & 0 & $.0 \%$ \\
& Regular & 8 & $19.5 \%$ \\
& Bueno & 20 & $48.8 \%$ \\
& Muy bueno & 11 & $26.8 \%$ \\
\cline { 2 - 4 } Cumplimiento del registro de & Muy malo & 0 & $.0 \%$ \\
Hoja Gráfica & Malo & 0 & $.0 \%$ \\
& Regular & 2 & $4.9 \%$ \\
& Bueno & 16 & $39.0 \%$ \\
Cumplimiento del registro de & Muy bueno & 23 & $56.1 \%$ \\
\cline { 2 - 4 } Balance Hídrico & Muy malo & 27 & $65.9 \%$ \\
& Malo & 0 & $.0 \%$ \\
& Regular & 2 & $4.9 \%$ \\
& Bueno & 12 & $29.3 \%$ \\
Kardex & Muy bueno & 0 & $.0 \%$ \\
\cline { 2 - 4 } & Muy malo & 0 & $.0 \%$ \\
& Malo & 0 & $.0 \%$ \\
& Regular & 14 & $34.1 \%$ \\
& Bueno & 23 & $56.1 \%$ \\
& Muy bueno & 4 & $9.8 \%$ \\
\hline
\end{tabular}

En la tabla 1 se muestra que las enfermeras del servicio de Ginecoobstetricia registran las Notas de Enfermería entre bueno y muy bueno en un $75.6 \%$, registran la Hoja Gráfica entre bueno y muy bueno en un $95.1 \%$, al igual que el Kardex en un $65.9 \%$. Mientras que el Balance Hídrico tiene un registro de muy malo $65.9 \%$.

\section{Tabla 2}

Nivel de cumplimiento general de los registros de Enfermería en el servicio de Ginecobstetricia del HONADOMANI "San Bartolomé". Lima-2012.

\begin{tabular}{cccc}
\hline Indicadores & Frecuencia & & $\%$ del N de la columna \\
\hline Total de cumplimento de los & Muy malo & 0 & $.0 \%$ \\
Registros de Enfermería & Malo & 0 & $.0 \%$ \\
& Regular & 15 & $36.6 \%$ \\
& Bueno & 23 & $56.1 \%$ \\
& Muy bueno & 3 & $7.3 \%$ \\
\hline
\end{tabular}

La tabla 2 muestra que las enfermeras del servicio de Ginecoobstetricia cumplen con realizar los registros de Enfermería en un $63.4 \%$ entre bueno y muy bueno y el $36.6 \%$ lo cumplen regularmente. No se evidencia el registro de malo o muy malo. 


\section{DISCUSIÓN}

La Joint Comission Acreditation of Health Care Organizations (JCAHO, 1991) recomienda el uso de indicadores clínicos que monitoricen la calidad y la adecuación de la atención en Enfermería. Asimismo, Ruiz y et al (2012) refieren que cada centro hospitalario debe construir sus propios modelos de registros de Enfermería, adaptándolos a sus necesidades y cumpliendo con las normas de la OMS. En el presente trabajo se aplicó una lista de verificación a una muestra de 41 historias clínicas de pacientes dados de alta, para conocer el nivel de cumplimiento en cada uno de los registros realizados por las enfermeras (anotaciones de Enfermería, control de funciones vitales, balance hídrico y Kardex) en los nuevos formatos validados y aplicados desde marzo del 2011 por el Comité de Historias Clínicas y el Departamento de Enfermería del HONADOMANI "San Bartolomé".

Chaparro L. y García (Colombia), Gonzales Itziar e Ibarra Beatriz y Corcoles Pilar (España), Méndez Gabriela y De los Santos (México) encontraron en sus estudios de investigación errores en los registros de Enfermería como un bajo cumplimiento de la normatividad: falta de datos de identificación, errores gramaticales, uso de corrector, borrones, abreviaturas no convencionales que inducen a error, líneas en blanco, firmas ilegibles y sin código; no hay consenso para registrar de forma estandarizada, ya que en cada servicio se realizan de diversas formas. No se encontró un trabajo de investigación específico sobre el cumplimiento de los registros de Enfermería. En el presente estudio se evaluó el nivel de cumplimiento de cada uno de los registros de Enfermería, se verificó el correcto llenado de cada uno de los ítems de cada formato, obteniéndose un $63.4 \%$ de registros entre bueno y muy bueno. Esto indica que aún se está en proceso de valorar la importancia de los registros de Enfermería y su implicancia en la atención del paciente.

En el año 2010, en el HONADOMANI "San Bartolomé", se realizó una auditoría de los Registros de Enfermería en las historias clínicas, siendo el nivel de cumplimiento en el servicio de Ginecoobstetricia de un $48 \%$, en general, no especificándose los ítems evaluados en cada registro. En la presente investigación realizada se observa que nivel de cumplimiento de los registros ha mejorado del año 2010 al 2012 en un 15.4\%; este avance contribuyó a optimizar el nivel de cumplimiento de los registros, convirtiéndose en un soporte documental donde queda plasmado la información sobre la actividad de la enfermera, referente a la valoración, diagnóstico, planeamiento, ejecución y evaluación incluyendo el tratamiento. Cumpliéndose con la Norma de la Reglamentación de la Ley del Trabajo del Enfermero $\mathrm{N}^{\circ} 27669$ el artículo $\mathrm{N}^{\circ} 9$ de "Brindar cuidado integral de Enfermería basado en el Proceso de Atención de Enfermería (PAE)".

El estudio realizado por Caballero, Becerra y Latrach (2001) demostraron que la mayoría del personal de Enfermería no aplica el proceso (PAE), porque no son factibles los métodos de registros. En el estudio se evidencia en las Notas de Enfermería que el $80.5 \%$ no realiza el registro de las evaluaciones de los objetivos, a pesar de que el formato incluye cada etapa del PAE, incumpliéndose en el registro las condiciones en las que se deja a la paciente y esto podría traer implicancias legales.

En cuanto al registro de las funciones vitales se encontró que el $4.9 \%$ no registra este dato: esta omisión podrían formar un riesgo para la evaluación correcta de los pacientes, pues como menciona (Fernández, Zárate, Ochoa, Ramírez, 2010; citado por Agustín 2008) el registro de los signos vitales permiten que la enfermera y el médico realicen una evaluación clínica confiable y tomen una decisión correcta y oportuna.

Chaparro L y García D. (2006) demostraron que el registro de mayor uso es la hoja de medicamentos, no refiriendo información sobre nivel de cumplimiento del registro. En el presente trabajo de investigación se encontró que solo el $85.4 \%$ trascribe correctamente las indicaciones médicas, el $90.2 \%$ no registra los datos relevantes (fecha de ingreso, procedencia, seguro y alergias), en el $73 \%$ de los registros la enfermera no cumple con firmar y sellar y en el $56.1 \%$ realiza el registro con borrones y manchas; estas deficiencias repercuten negativamente en el tratamiento del paciente por omitir la terapia indicada y demuestra una irresponsabilidad en la práctica de la profesión que puede conllevar a problemas ético-legales. En el presente estudio se encontró que el $65.9 \%$ tienen un registro de kardex entre bueno y muy bueno donde se incluyen el tratamiento médico, sin embargo un $34.1 \%$ tiene un registro regular lo que indica que está desviando datos importantes necesitando concientizar su relevancia en la gestión de cuidado.

En los resultados del trabajo de investigación se evidenció que el $34.1 \%$ registra los ingresos, el $29.3 \%$ los egresos y solo el $26.8 \%$ realiza el balance hídrico total. Ortega y Suárez (2009) menciona con respecto a la hidratación parenteral completa, que las enfermeras registran el inicio de la vía venosa y la cantidad de solución indicada; no siempre la hora de término, 
ni la cantidad de líquido que se había administrado en total; dedujo que no se hacía balances, ya que lo que no está escrito no está realizado. Situación preocupante que refleja que la enfermera no está consciente de la importancia de este registro que evalúa el equilibrio hidroelectrolítico de la paciente, ya que en base a este resultado, se tomarán nuevas decisiones para el tratamiento.
Se concluye que el más de $50 \%$ de las enfermeras del servicio de Ginecoobstetricia cumplen con realizar los registros de enfermería en un nivel bueno y muy bueno y un $36.6 \%$ tiene un llenado regular.

\section{REFERENCIAS BIBLIOGRÁFICAS}

Agustín, A. (2008). Aplicación del método cubano de registro clínico del Proceso de Atención de Enfermería. (Tesis doctoral). Ministerio de Salud Pública, Hospital Clínico Quirúrgico "Hermanos Amejeiras". La Habana - República de Cuba. Recuperado de: http://tesis.repo. sld.cu/42/1/Leon-Roman-CA.pdf

Avendaño, M., Ramírez, T., Colín, L., Rentería, C., y López, C. (2011). Exactitud, precisión y curva de aprendizaje del instrumento para los registros clínicos de enfermería. Estudio comparativo. Revista de Especialidades Médico-Quirúrgica. 16 (2), 97-103. Recuperado de: http://www.redalyc.org/pdf/473/47319326008.pdf

Becerra R, Caballero E. (2006). Instrumentación de la gestión clínica intraoperatoria mediante un registro electrónico. Santiago de Chile: Eds. Universidad Católica.

CEP (2006). Código de ética y deontología de enfermería. Lima - Perú. Recuperado de: http://www.cep.org.pe/ beta/download/codigo_etica_deontologia.pdf

CEP(2002). Ley del trabado del enfermero de la Enfermera(o) $N^{o}$ 27669. Lima - Perú. Recuperado de: http://www.cep. org.pe/beta/download/ley_trabajo_enfermero.pdf

Caballero, E., Becerra, R., Latrach, C. (2005). Grado de uso del proceso de Enfermería. Santiago de Chile: Eds. Universidad Católica.

Corcoles, P., García, P., Ruiz, T., Pita, L., \& Callejas, C. (2002). El dossier de enfermería en el complejo hospitalario de Albacete: Composición y control de calidad. Revista Enfermería. 7(207),1-3. Recuperado de:http://www.uclm.es/ab/enfermeria/revista/ numero\%207/dossier7.htm

Chaparro, L. (2003). Registros del Profesional de Enfermería como evidencia de la continuidad del cuidado. Rev. Actual Enferm. 6(4), 16-21.

García, E. (1997). Monitorización y Evaluación de las actividades de la calidad. La calidad aplicada a los cuidados de Enfermería. Cádiz: Edit. Colegio Oficial de Diplomado de Enfermería.

García, S., y Meza, A. (2003). Análisis de los Informes de Enfermería de los Hospitales Públicos de la ciudad de Corrientes. Argentina: Universidad Nacional del Nordeste. Recuperado de: http://www.unne.edu.ar/ unnevieja/Web/cyt/cyt/2003/comunicaciones/03Medicas/M-028.pdf
García, R., Navío, A., Valentín, L. (2007). Normas básicas para la elaboración de los registros de enfermería. Nure Investigación. 1(28), 1-8. Recuperado de: http:// www.nureinvestigacion.es/ficheros_administrador/ protocolo/pdf_protocolo28.pdf

González, J. (1999). Historia de la enfermería. España: Editorial Dialnec

Hernández, D., Rodríguez, M., Gutiérrez, \& M., Bolaños, G. (2007). Elaboración de un registro enfermero para hemodiálisis según la norma ISO 9001:2005. Rev. Soc. Esp. Enferm. Nefrol. 10(1),5-22. Recuperado en:http://scielo.isciii.es/scielo.php?script=sci_ arttext\&pid $=\mathrm{S} 1139-13752007000100003 \& \operatorname{lng}=\mathrm{es}$. http://dx.doi.org/10.4321/S1139-13752007000100003.

Junttila, K., Salantera, S., Hupli, M. (2000). Perioperative documentation in Finland. AORN Journal. 72(5), 862-864. Recuperado de: http://www.aornjournal.org/ article/S0001-2092(06)62018-8/abstract

Joint Comission on Acreditation of Health Care Organizations (1991). The revised JCAHO nursing care standards: areas of emphasis. Joint Crit Care Nurse. 11(8),12,14. Recuperado de: http://www.ncbi.nlm.nih. gov/pubmed/1893757

Loza, H, Vázquez, V. (2005). Impacto de la implementación del proceso de Enfermería en el INNS, mediante una prueba piloto. Rev. Enf. Neurol. 4(1),1-6.

Ortega, C. Suárez, M., (2009). Manual de evaluación de la calidad del servicio de servicio de Enfermería: Estrategia para su aplicación. México: Editorial Médica Panamericana S.A.

Potter, P., Perry, A. (2005). Fundamentos de Enfermería. Madrid: Elsevier.

Koutoukidis, G., Stainton, K., y Hughson, J. (2008). Tabbner Nursing Care. Australia: Editorial Elsevier

Ruiz, A. y et al (2012). Manual de Procedimientos generales de Enfermería. Sevilla:Hospital Universitario Virgen del Rocío. Servicio Andaluz de Salud. Recuperado de: http:// www.juntadeandalucia.es/agenciadecalidadsanitaria/ observatorioseguridadpaciente/gestor/sites/ PortalObservatorio/es/galerias/descargas/recursos compartidos/procedimientos_generales_enfermeria_ HUVR.pdf 